\title{
Reproductive pattern in the solanum mealybug, Phenacoccus solani: A new perspective
}

\author{
Jun Huang ${ }^{\text {Corresp., } 1}$, Fuying Zhi ${ }^{1,2}$, Juan Zhang ${ }^{3}$, Muhammad Hafeez ${ }^{1}$, Xiaowei Li ${ }^{1}{ }^{1}$ Jinming Zhang ${ }^{1}$, Zhijun Zhang $^{1}$, \\ Likun Wang ${ }^{1}$, Yaobin lu ${ }^{\text {Corresp. } 1}$ \\ 1 Institute of Plant Protection and Microbiology, Zhejiang Academy of Agricultural Sciences, Hangzhou, China \\ 2 College of Chemistry and Life Sciences, Zhejiang Normal University, Jinhua, China \\ 3 Institute of Garden Plants and Flowers, Zhejiang Academy of Agricultural Sciences, Hangzhou, China \\ Corresponding Authors: Jun Huang, Yaobin lu \\ Email address: junhuang1981@aliyun.com, luybcn@163.com
}

Background. The reproductive pattern of most scale insects is ovoviviparity. The solanum mealybug, Phenacoccus solani (Hemiptera: Pseudococcidae), is known as a thelytokous parthenogenetic species, but there is still debate about the reproductive strategies of this species.

Methods. Here, we investigated the oviposition characteristics of $P$. solani and used scanning/transmission electron microscopy and RNA-seq to identify the differences between two types of eggs.

Results. We found that $P$. solani laid two types of eggs in one batch, with no significant difference in apparent size: one with eyespots that hatched and another without eyespots that failed to hatch. Furthermore, the physiological and molecular differences between the two types of eggs were highly significant. KEGG enrichment analysis revealed significant enrichment for the JAK-STAT, Notch, Hippo, and Wnt signaling pathways and dorsoventral axis formation, wax biosynthesis, cell cycle, insulin secretion, and nitrogen metabolism pathways. The results suggest that the embryo of the egg undergoes development inside the mother and only a short molting period outside the mother.

Discussion. Ovoviviparous species produce eggs and keep them inside the mother's body until they are ready to hatch, and the offspring exits the egg shell during or immediately following oviposition. Therefore, we suggest that the reproductive pattern of $P$. solani can be described as ovoviviparity. 


\section{Reproductive pattern in the solanum mealybug,}

\section{Phenacoccus solani: A new perspective}

3 Jun Huang ${ }^{1}$, Fuying Z Zij ${ }^{1,2}$, Juan Zhang ${ }^{3}$, Muhammad Hafeez ${ }^{1}$, Xiaowei Li ${ }^{1}$, Jinming

4 Zhang ${ }^{1}$, Zhijun Zhang ${ }^{1}$, Likun Wang ${ }^{1}$, Yaobin LU ${ }^{1}$

5

$6{ }^{1}$ Institute of Plant Protection and Microbiology, Zhejiang Academy of Agricultural

7 Sciences, Hangzhou 310021, China;

$8{ }^{2}$ College of Chemistry and Life Sciences, Zhejiang Normal University, Jinhua 321004,

9 China;

$10{ }^{3}$ Institute of Garden Plants and Flowers, Zhejiang Academy of Agricultural Sciences,

11 Hangzhou 311202, China;

12 Corresponding Author:

13 Jun Huang

14 NO. 298, Desheng Road, Hangzhou City, Zhejiang Province, 310021, China.

15 Junhuang1981@aliyun.com

16 Yaobin LU

17 NO. 298, Desheng Road, Hangzhou City, Zhejiang Province, 310021, China.

18 luybcn@163.com 


\section{Abstract}

33 Background. The reproductive pattern of most scale insects is ovoviviparity. The

34

35

36

53

57

58

59

60

61

62

63 solanum mealybug, Phenacoccus solani (Hemiptera: Pseudococcidae), is known as a thelytokous parthenogenetic species, but there is still debate about the reproductive strategies of this species.

Methods. Here, we investigated the oviposition characteristics of $P$. solani and used scanning/transmission electron microscopy and RNA-seq to identify the differences between two types of eggs.

Results. We found that $P$. solani laid two types of eggs in one batch, with no significant difference in apparent size: one with eyespots that hatched and another without eyespots that failed to hatch. Furthermore, the physiological and molecular differences between the two types of eggs were highly significant. KEGG enrichment analysis revealed significant enrichment for the JAK-STAT, Notch, Hippo, and Wnt signaling pathways and dorsoventral axis formation, wax biosynthesis, cell cycle, insulin secretion, and nitrogen metabolism pathways. The results suggest that the embryo of the egg undergoes development inside the mother and only a short molting period outside the mother.

Discussion. Ovoviviparous species produce eggs and keep them inside the mother's body until they are ready to hatch, and the offspring exits the egg shell during or immediately following oviposition. Therefore, we suggest that the reproductive pattern of $P$. solani can be described as ovoviviparity.

Subjects Agricultural Science, Entomology, Insect Bioecology Keywords mealybug, reproductive pattern, oviposition, egg, ovoviviparity, Phenacoccus solani 


\section{Introduction}

65 The reproductive strategies of most insects have been described with three main 66 patterns: oviparity, viviparity and ovoviviparity (Meier et al., 1999; Wheeler, 2003;

67 Gullan \& Granston, 2014). In oviparous species, egg development occurs in the

68

69

70

71

72

73

74

75

76

77

78

79

80

81

82

83

84

85

86

87

88

89

90

91

92

93

94

external environment after oviposition, and hatching occurs outside the mother's body, whereas in viviparous species (which are relatively rare among insects), egg development occurs inside the mother's body, which provides gas exchange and, more importantly, nourishment for the embryos which are born alive (Andrews \& Rose, 1994; - -Tworzydlo et al., 2013). Based on nutritional relationships between maternal and embryonic organisms, two modes of viviparity are recognized: matrotrophic and lecidotrophic (Ostrovsky et al., 2016). Ovoviviparity is in fact a specific type of viviparity where developing eggs are retained within the body of the mother, and the offspring are nourished by the reserve materials accumulated in the eggs during oogenesis (Blackburn, 1999; Gaino \& Rebora, 2005; Lodé, 2012). Therefore, the recent view is that there are truly only two main reproductive strategies: viviparity and oviparity (Ostrovsky, 2013; Ostrovsky et al., 2016). In previous studies, some insects, such as cockroaches (Warnecke \& Hintze-Podufal, 1996), aphids (Ortiz-Rivas et al., 2004), tsetse flies (Meier et al., 1999), thrips (Kranz et al., 2002), and scale insects (Gavrilov \& Kuznetsova 2007; Ngernsiri et al., 2015), were described as ovoviviparous species. Scale insects (Hemiptera: Sternorrhyncha: Coccoidea), like many Hemiptera, feed on sap drawn directly from the plant vascular system and secrete a waxy coating for defense; in addition, many scale insect species are major quarantine pests of agricultural or ornamental plants in tropical/subtropical climates as well as in greenhouses in temperate zones worldwide (Gullan \& Kosztarab 1997; Gavrilov-Zimin, 2018). All previous relevant studies suggest that the phenomenon of ovoviviparity is widely distributed among scale insects (Tremblay, 1997; Gavrilov \& Kuznetsova, 2007). Gavrilov-Zimin (2018) provided an overview of the distribution of different variants of ovoviviparity/viviparity among scale insect families and demonstrated that the evolution of scale insects shows multiple cyclic conversions of the oviparous reproduction pattern to ovoviviparity/viviparity, with the appearance of new and interesting adaptations for egg protection. In other words, the reproductive modes of scale insects may be rich and 
95

96

97

98

99

100

101

102

103

104

105

106

107

108

109

110

111

112

113

114

115

116

117

118

119

120

121

122

123

124 125

variable; however, the understanding of the course of evolution of reproductive patterns in these insects is not complete (Gavrilov \& Kuznetsova, 2007). Therefore, the identification of reproductive patterns has great heuristic value in terms of both reproductive and evolutionary biology.

The solanum mealybug, Phenacoccus solani (Hemiptera: Pseudococcidae), is native to North America (Chatzidimitriou et al., 2016) and is a newly recorded species in China. Furthermore, this mealybug species is quite polyphagous and considered to be a major threat to agricultural production and the ecological environment, causing significant problems (Ben-Dov, 2005a; Zhi et al., 2018). P. solani is a thelytokous parthenogenetic species, and no male individuals are found in its populations (Lloyd, 1952; Ben-Dov, 2005b; Zhi et al., 2018). Regarding the birth strategies of $P$. solani, McKenzie reported that this species was viviparous (McKenzie, 1967); however, Kosztarab (1996) and Chatzidimitriou et al. (2016) considered this species to be ovoviviparous. Vennila et al. (2010) found that in another species of mealybug in the same genus, parthenogenesis via ovoviviparity $(96.5 \%)$ was dominant over oviparity (3.5\%). Many scholars have found that the hatching period of eggs laid by mealybugs is very short and that the hatching process is relatively concealed (beneath the abdomen), as a result, short and concealed hatching has been suggested as the main reason for the divergence of reproductive modes in mealybugs (Tremblay, 1997; Lagowska \& Golan, 2009; Vennila et al., 2010; Zhi et al., 2018). According to our previous observations, we believe that the reproductive mode in mealybugs, at least in $P$. solani, is complex and not simple to define.

Here, we investigated the oviposition characteristics of $P$. solani and used scanning electron microscopy (SEM) and transmission electron microscopy (TEM) to distinguish between two types of eggs (i.e., reproductive products putatively considered to be eggs) laid in one batch. Finally, we further investigated miRNA and mRNA expression in the two types of eggs. We aimed to answer the following questions: 1) What life stage is $P$. solani laying - an egg, a nymph, or something in between? 2) If different reproductive "products" coexist, do they differ in appearance, physiology and molecular biology? 3) According to the above findings, what is the reproductive mode of $P$. solani? 


\section{Materials \& Methods}

\section{Oviposition characteristics}

128 The oviposition process of female adults of $P$. solani was observed on two transparent, 129 single-well, concave slides (Fig. 1) (length * width $=76.2 \mathrm{~mm}$ * $25.4 \mathrm{~mm}$; the thickness

130 of each concave slide was $1.3 \mathrm{~mm}$; the diameter and depth of the circular hole were 15 $131 \mathrm{~mm}$ and $0.5 \mathrm{~mm}$, respectively). The steps of the procedure were as follows: 1) a 132 concave slide was placed face up on a flat table and a female adult was gently placed 133 into the middle of the hole of the concave slide with a brush; 2) quickly another concave 134 slide was placed face down, on top of the bottom concave slide; and 3) finally, rubber 135 bands were used to bind the ends of the two concave slides to secure them. Preliminary 136 experiments showed that the thickness of female adults was $0.91 \pm 0.03 \mathrm{~mm}$, so the 137 above observation method did not harm the bodies of female adults. The egg hatching 138 process was further tracked and observed under a NikonSMZ1500 zoom stereomicroscope (Nikon, Japan), and photographs were taken every 5 minutes from the start of the egg laying process.

142 The number, length, width and hatching rate of eggs with eyespots (hereafter eggs withES) or eggs without eyespots (hereafter eggs without-ES) were further observed in the same batch of eggs under a NikonSMZ1500 zoom stereomicroscope. There were two kinds of treatments: one employed the concave slide method (placing a female adult between two concave slides as mentioned above), and the other employed the blade method, i.e., placing a female adult on detached potato leaves, with the petiole wrapped with defatted cotton to maintain leaf freshness and the placing the whole treated leaves in Petri dishes (diameter $=9 \mathrm{~cm}$, thickness $=1.4 \mathrm{~cm}$ ). The oviposition of female adults was observed every 30 minutes from 9.00 a.m. to 4.00 p.m, and the numbers of the two kinds of eggs were counted. Each female adult was biometrically tested once, and each treatment was repeated 15 times. A total of 25 eggs were randomly selected from the two kinds of eggs, and their lengths and widths were measured. After 72 hours, the hatching of the two kinds of eggs was observed. Eggs with-ES hatched 114 individuals, while eggs without-ES hatched 45 individuals. The experiment was carried out in an 
156 artificial climate chamber with a temperature of $27 \pm 1{ }^{\circ} \mathrm{C}$, a humidity of $70 \% \pm 5 \%$ and a 157 photoperiod of $16 \mathrm{~L}: 8 \mathrm{D}$.

158 Does the mother's body affect the hatching of eggs?

159 Two treatments were established: 1) eggs with-ES were incubated under the mother's 160 body (Fig. 2A), and 2) eggs with-ES were artificially removed from the mother and kept 161 on the concave slide (Fig. 2C). Then, the female adults were continuously observed 162 through a NikonSMZ1500 zoom stereomicroscope every five minutes. Continuous 163 stretching of the abdomen by female adults indicated that they were about to lay eggs. 164 For treatment 2, the upper concave slide was removed immediately, and the mother 165 was carefully removed with an insect pin. Hatching time was recorded as the time from 166 when the female adult laid eggs to the time when the eggshell was completely 167 detached. Each egg was bioassayed once; experiment 1 included 30 replicates, and 168 experiment 2 included 47 replicates.

\section{Microscopic differences between the two kinds of eggs}

\section{Egg surface and internal structure}

171 The surface and internal structure of the two kinds of eggs were observed by SEM 172 (SU8010, Hitachi, Japan) and TEM (H7650, Hitachi, Japan). The collected eggs with 173 and without ES were pretreated with liquid nitrogen immediately. The follow-up 174 procedures followed the instrument operation methods for the scanning electron 175 microscope. TEM was performed as follows: 1) fixation, eggs were immersed in $2.5 \%$ 176 glutaraldehyde fixative solution and then rinsed with buffer solution; 2) gradient 177 dehydration, the fixed samples were dehydrated with an ethanol; 3) gradient osmosis, 178 the samples were permeated with a mixture of acetone and Spurr resin penetrant (1:1); 179 4) embedding and polymerization, $100 \%$ Spurr embedding agent was added, followed 180 by polymerization for 24 hours; 5 ) ultrathin sectioning, the samples were cut to approximately $90 \mathrm{~nm}$; and 6) observation and photography.

\section{RNA sequencing and data analysis}

RNA extraction, library construction and RNA sequencing

Samples of newly laid eggs (with-ES or without-ES) were collected and immediately placed in a 0.5 MIEP tube and then frozen in liquid nitrogen for RNA extraction. Total 
187 described (Yin et al., 2018). The libraries were sequenced on the Illumina HiSeq X Ten 188 platform, and 150 bp paired-end reads were generated. Raw data (raw reads) in fastq 189 format were first processed using Trimmomatic (Bolger et al., 2014). Reads containing 190 poly-N stretches and reads oflow quality were removed to obtain clean reads. After 191 removing adaptor and low-quality sequences, the clean reads were assembled into 192 expressed sequence tag clusters (contigs) and de novo assembled into transcripts by 193 Trinity (Grabherr et al., 2011) (version: 2.4) with the paired-end method. The longest 194 transcript was chosen as a unigene based on similarity and length for subsequent 195 analyses. Raw data were deposited in the National Center for Biotechnology 196 Information (NCBI) Sequence Read Archive (SRA) (https://www.ncbi.nlm.nih.gov/sra) under accession number PRJNA554708. Unigene de novo assembly, functional annotation and data analysis Transcriptome sequencing and analysis were conducted by OE Biotech Co., Ltd. (Shanghai, China). The functions of the unigenes were annotated by alignment of the 201 unigenes with the NCBI nonredundant (NR), SwissProt, EuKaryotic Orthologous Groups 202 (KOG), Gene Ontology (GO) and Kyoto-Encyclopedia of Genes and Genomes (KEGG) 203 databases. Differentially expressed unigenes (DEGs) were identified using the DESeq (Anders \& Huber, 2013) functions "estimate size factors" and "nbinom test". A $p$ value < 0.05 and fold change $>2$ or $<0.5$ were set as the thresholds for significant differential 206 expression. Hierarchical cluster analysis of DEGs was performed to explore transcript expression patterns, and KEGG pathway enrichment analysis of DEGs was performed using $R$ based on a hypergeometric distribution.

\section{Results}

\section{Oviposition characteristics}

212 Female adults of $P$. solani produced eggs in one generation through thelytokous parthenogenesis, and the eggs were long and oval, similar to those described by Zhi et al. (2018). Female adults secreted silken wax, but it never formed an ovisac. After egg laying, the eggshell was detached from the nymph, followed by the appearance of $1^{\text {st }}$

216 instar nymphs that quickly crawled away from the lower part of the mother. Moreover, in

217 a batch of eggs laid by female adults of $P$. solani, two kinds of eggs with distinct 
218 morphological differences appeared (Fig. 3A/B): two reddish-brown eyespots were seen 219 on one type of egg (eggs with-ES; Fig. 3D), while the other type did not display these 220 eyespots (eggs without-ES; Fig. 3C). The hatching process of eggs with-ES was as 221 follows: 1) in the first 5 minutes, the eggs began to show considerable peristalsis 222 beneath the mother, and at 10-15 minutes, the eggshell was gradually detached, and 223 wax powder appeared on the surface of the body; and 2) at approximately 20 minutes,

224 antennae and feet began to appear, and the hatching process was generally completed 225 within 25 minutes, while the detached eggshell could be seen at the abdominal end of 226 the 1st instar nymph (Fig. 4).

\section{Egg morphology}

228

229

230

231

232

233

234

235

236

237

238

239

240

241

242

243

244

245

246

247

248

In the present study, we observed that female adults could lay two types of eggs, those with-ES and those without-ES, in either treatment. The ratio of eggs with-ES was significantly larger than that of eggs without-ES (on concave slides, $91.56 \pm 2.14$ vs. $8.44 \pm 2.14$, respectively, $\chi 2=86.01, \mathrm{n}=15, p<0.001$; on leaves, $87.31 \pm 2.90$ vs. $12.69 \pm 2.90$, respectively, $\chi 2=13.07, n=15, p<0.001$ ) (Fig. 5A). The eggs were long and elliptical, with lengths of $0.320 \pm 0.006 \mathrm{~mm}$ (with-ES) and $0.305 \pm 0.008 \mathrm{~mm}$ (without-ES) ( $\mathrm{t}=1.42, \mathrm{n}=25$; Fig. $5 \mathrm{C}$ ) and widths of $0.146 \pm 0.002 \mathrm{~mm}$ (with-ES) and $0.147 \pm 0.003 \mathrm{~mm}$ (without-ES) $(t=0.24, \mathrm{n}=25 ;$ Fig. $5 \mathrm{D}$ ). Furthermore, the hatching rate of eggs with-ES was $100 \%$, and no eggs without-ES hatched (Fig. 5B).

\section{Does the mother's body affect the hatching of eggs?}

To investigate the extent to which the mother's body affected the hatching of eggs, eggs without-ES were removed from the area beneath the mother's body after they were laid. Therefore, we further tested whether eggs with-ES hatched after they were removed from the area beneath the mother's body. We found that eggs with-ES could hatch normally under any treatment (Fig. $2 \mathrm{~A} / \mathrm{B} / \mathrm{C}$ ), but the hatching times were different. Inside the mother's body, the hatching time was $24.30 \pm 0.60$ minutes (Fig. 2D), but when the mother's body was removed, the hatching time was reduced by nearly 5 minutes to $19.47 \pm 0.45$ minutes (Fig. $2 \mathrm{E}$ ). Therefore, we suggest that the mother's body has no effect on the success of egg hatching, or it could be inferred that the failure of eggs without-ES to hatch was largely due to internal factors. Many species of scale insects secrete abundant wax and form an ovisac that covers eggs and prevents their 
249 adhesion, but in some species, ovisacs are not built, and the time of egg development 250 outside of the maternal body is decreased (Gavrilov-Zimin, 2018). P. solani belongs to 251 the latter group, secreting a small amount of wax and never forming an ovisac. Thus, it 252 is not surprising that the eggs of $P$. solani hatched so quickly, especially when the eggs 253 were isolated.

\section{Microscopic differences between the two types of eggs}

2551 Egg surface and internal structure

256 The surface and internal structure of eggs with and without-ES were observed using 257 SEM and TEM. The contour of appendages could be clearly seen across the eggshell, 258 and the bristles, tubular glands and six conical receptors (which were symmetric, with 259 three on each side) could be seen on the surfaces of eggs with-ES (Fig. 6A); moreover, 260 complete blood cells, cytoplasm, mitochondria and myocutaneous filaments were 261 observed inside (Fig. 6B). Eggs without-ES had a smooth surface (Fig. 6C) and 262 contained only lipid droplets, endoplasmic reticulum and free ribosomes (Fig. 6D). 2

263 RNA sequencing and data analysis

264 Illumina sequencing generated approximately $45 \mathrm{M}$ reads per sample after the removal 265 of low-quality reads. These reads were assembled randomly and produced 55,558 266 unigenes with an N50 of 1,026 nt. After annotating unigenes with several databases and 267 calculating the expression of unigenes as fragments per kilobase of exon model per 268 million reads mapped (FPKM), correlation coefficients between samples were 269 calculated and used to estimate biological repeatability and differences between groups. 270 The correlation coefficient of 3 biological replicates in the group with-ES and the group 271 without-ES was $>0.8$, and the sample correlation coefficient between these two groups 272 was only 0.4 , showing an obvious difference between these two groups (Fig. 7A). DEGs 273 were identified and screened with the thresholds of $p<0.05$ and fold change $>2$ (or fold 274 change < 0.5) $(\mathrm{Fig} .7 \mathrm{~B} / \mathrm{C})$. There were 13,164 DEGs between the with-ES and without275 ES groups, including 9,243 up regulated DEGs and 3,921 down regulated DEGs. The 276 DEGs are shown with a volcano plot, and a heat map was generated from hierarchical 277 cluster analysis to show the expression patterns of the DEGs (Fig. 7B). KEGG 278 enrichment analysis of the DEGs was performed to determine the main pathways 279 associated with these DEGs. We found that the JAK-STAT, Notch, Hippo, and Wnt 
280 signaling pathways and dorso-ventral axis formation, wax biosynthesis, progesterone-

281 mediated oocyte maturation, cell cycle, eukaryote ribosome biogenesis, insulin

282 secretion, and nitrogen metabolism pathways were significantly enriched (Fig. 8).

283

\section{Discussion}

\section{Oviposition and hatching characteristics}

286

287

288

289

290

291

292

293

294

295

296

297

298

299

300

301

302

303

304

305

306

307

308

309

310

In the present study, we found eggs laid by parthenogenetic $P$. solani, but soon emerged $1{ }^{\text {st }}$-instar nymphs following hatch. Because this hatching process is fast $(<30$

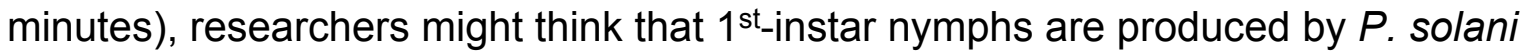
female adults. A similar phenomenon was reported in another invasive mealybug, Phenacoccus solenopsis, and because both eggs and 1 st-instar nymphs were found in oocysts, Vennila et al. (2010) considered the scale insect to be able to reproduce in both ways, i.e., via ovoviviparity and oviparity. In Coccus hesperidum, naked nymphs appeared from the vulvar orifice, but thin eggshells were shown to remain in the female reproductive tract (Hagan, 1951), and in $P$. solani, the eggshells were kept beneath the mother.

In Matsucoccus matsumurae, there are two types of eggs, those with and without eyespots, but the eggs with eyespots are similar to those without eyespots, and the egg types show different developmental periods with normal hatching (Xie et al., 2014). However, $P$. solani laid two types of eggs in one batch, with no significant difference in apparent size: one with eyespots that hatched and another without eyespots that failed to hatch. Generally, eggs are under stress from external factors, which may prevent them from hatching properly. For example, some of the eggs laid by heat-treated females of Nilaparvata lugenswere unable to hatch due to failure during blastokinesis (Lee \& Roger, 1987). Further research revealed that yeast-like symbiotes in N. lugens play an important role in the embryonic and postembryonic development of eggs, especially the ventral differentiation of the embryo (Wilkinson \& Ishikawa, 2001; Nan et al., 2016). The symbiotic bacteria in the bodies of most mealybug subfamily insects are Tremblaya princeps (Gruwell et al., 2010), play a substantive role in the host plant specificity of their hosts (Baumann, 2005; Hansen \& Moran, 2014), and are correlated with host development (Huang et al., 2015); therefore, we suggest that the absence of 
311 symbiotes might explain the presence of nondeveloping eggs. Another type of insect

312 egg that does not hatch is the nutritive egg common in social insects, such as ants. The 313 nutritive eggs of ants are unfertilized eggs that cannot hatch and are eaten in colonies 314 containing a queen (Heinze et al., 1996, 1999). However, for this parthenogenetic and 315 thelyotokous species of mealybug, the factors causing $P$. solani to lay eggs that cannot 316 hatch require further study.

\section{Physiological and molecular differences between the two types of eggs}

318 According to these observations of egg surface and internal structure, we suggest that 319 eggs with-ES are alive, with features such as conical receptors, blood cells and 320 myocutaneous filaments, and that they still have an eggshell and are close to hatching, even though they no longer resemble an egg on a microscopic level. Although some important organelles such as endoplasmic reticulum and free ribosomes were present in the eggs without-ES, mitochondria had never been found. Mitochondria are a special organelle that contain their own genomes and plays an important role in oocyte maturation and embryo development (Ferenz, 1993; Lieber et al., 2019). Moreover, mitochondria are inherited only in the maternal line, i.e., mitrochondrial DNA is passed only through the mother's egg cell (Ma et al., 2014; Lieber et al., 2019). Lack of mitochondrial redistribution in cytoplasm was a sign of immature oocyte and was closely related to low developmental of eggs (Bavister \& Squirrell, 2000). If mitochondria were missing, it could be fatal to the development of egg cell and later embryonic development. Therefore, we hypothesized that the development of egg cell might be arrested for the eggs without-ES in the absence of mitochondria.

We further determined the differences between eggs with and without ES at the molecular level. The result revealed that the differences in terms of unigene expression between two types of eggs were highly significant, and we also found that the JAKSTAT, Notch, Hippo, and Wnt signaling pathways and some important pathways related to metabolism and nutrition were significantly enriched. Recently, the JAK-STAT, Notch, Hippo and Wnt signaling pathways were found to independently or interactively participate in the regulation of egg production (Hombria \& Brown, 2002; McGregor et al., 2002). For example, mutual antagonism between the Notch and JAK/STAT signaling pathways provides a crucial facet of follicle cell patterning and ultimately helps establish 
342 the polarity of the egg chamber (Assa-Kunik et al., 2007), and the Hippo pathway

343 controls polar cell specification by repressing Notch activity (Chen et al., 2011).

344 Moreover, some of these important signaling pathways are involved in aspects of cell

345 development and metabolic function, such as dorsal-ventral axis formation, wax

346 biosynthesis, insulin secretion and nitrogen metabolism.

347

\section{Conclusions}

349

350

351

352

353

354

355

356

357

358

359

360

361

362

363

364

365

366

367

368

369

370

371

372

\section{4}

Although the reproductive mode of $P$. solani has been described previously, there is still no clear agreement on its definition. We found no differences between the two types of eggs by visual observation, but the physiological and molecular differences were highly significant. The results suggest that the embryonic development of eggs with-ES is complete when the eggs are laid beneath the abdomen, i.e., the embryo of the egg develops inside the mother. However, the embryonic development of eggs without-ES seems to be incomplete. Although there are no direct data on the entire process of embryonic development, we can at least be sure that the cell development and physiological metabolism of eggs without-ES are hindered or arrested.. Moreover, we found that eggs with-ES begin to hatch and shed their eggshell (immediately) after leaving the mother's body: i.e., this species lays eggs and does not experience live birth. Ovoviviparous species oviposit eggs at an advanced stage of embryological development, and the larva exits the eggshell during or immediately following oviposition (Meier et al., 1999). Therefore, we suggest that the reproductive pattern of $P$. solani can be described as ovoviviparity.

\section{Acknowledgements}

We would like to thank He Ziyi (Senior Experimentalist, Nanjing Agricultural University), and Liao Zhenfeng (Zhejiang Academy of Agricultural Sciences) for their support of electron microscope samples making and photos taking, and Dr. Wenya Lu and Dr. Fan Zhang (OE Biotech, Shanghai, China) for assistance with the bioinformatics analysis of the sequencing data. We are so grateful to Zhou Zhongshi (Chinese Academy of Agricultural Sciences), Jiang Mingxing (Zhejiang University), Xu Yijuan (South China 
373 Agricultural University), Ma Jun (Guangzhou Customs), and Fu Jianwei (Fujian

374 Academy of Agricultural Sciences) for their comments and suggestions on the 375 manuscript.

376

377

378

379

380

381

382

383

384

385

386

387

388

389

390

391

392

393

394

395

396

397

398

399

400

401

402

403

\section{References}

Andrews RM, Rose BR. 1994. Evolution of viviparity: constrains on egg retention. Physiol Zool. 67, 1006-1024 DOI 10.2307/30163876.

Anders S, Huber W. 2013. Differential expression of RNA-Seq data at the gene levelthe DESeq package. European Molecular Biology Laboratory, Heidelberg, Germany.

Assa-Kunik E, Torres IL, Schejter ED, Johnston DS, Shilo BZ. 2007. Drosophila follicle cells are patterned by multiple levels of Notch signaling and antagonism between the Notch and JAK/STAT pathways. Development, 134, 1161-1169 DOI 10.1242/dev.02800.

Baumann P. 2005. Biology of bacteriocyte-associated endosymbionts of plant sapsucking insects. Annu Rev Microbiol. 59,155-189 DOI 59.030804.121041.

Bavister BD, Squirrell JM. 2000. Mitochondrial distribution and function in oocytes and early embryos. Hum Reprod. 5, 189-198 DOI 10.1093/humrep/15.suppl_2.189.

Ben-Dov Y. 2005a. A systematic catalogue of the scale insect Family Margarodidae (Hemiptera: Coccoidea) of the World. Publisher Intercept Scientific Technical Medical. London. 400.

Ben-Dov Y. 2005b. The solanum mealybug, Phenacoccus solani Ferris (Hemiptera: Coccoidea: Pseudococcidae), extends its distribution range in the Mediterranean Basin. Phytoparasitica. 33, 15-16 DOI 10.1007/BF02980920.

Blackburn DG. 1999. Viviparity and oviparity: evolution and reproductive strategies. In book: Encyclopedia of Reproduction Vol. 4, Publisher: Academic Press, London (Editors: Knobil E, Neill JD). 994-1003.

Bolger AM, Lohse M, Usadel B. 2014. Trimmomatic: a flexible trimmer for Illumina sequence data. Bioinformatics. 30, 2114-2120.

Chatzidimitriou E, Simonato M, Watson GW-, Martinez-Sanudo I, Tanaka H, Zhao J, Pellizzari G. 2016. Are Phenacoccus solani Ferris and P. defectus Ferris 
404

405

406

407

408

409

410

411

412

413

414

415

416

417

418

419

420

421

422

423

424

425

426

427

428

429

430

431

432

433

434

(Hemiptera: Pseudococcidae) distinct species? Zootaxa. 4093, 539-551 DOI 10.11646/zootaxa.4093.4.5.

Chen HJ, Wang CM, Wang TW, Liaw GJ, Hsu TH, Lin TH, Yu JY. 2011. The Hippo pathway controls polar cell fate through Notch signaling during Drosophila oogenesis. Dev Biol. 357, 370-379 DOI 10.1016/j.ydbio.2011.07.003.

Ferenz HJ. 1993. Yolk protein accumulation in Locusta migratoria (Orthoptera: Acrididae) oocytes. Int J Insect Morphol Embryol. 22, 295-314 DOI: 10.1016/00207322(93)90016-T.

Gaino E, Rebora M. 2005. Egg envelopes of Baetis rhodani and Cloeon dipterum (Ephemeroptera, Baetidae): a comparative analysis between an oviparous and an ovoviviparous species. Acta Zool. 86, 63-69 DOI 10.1111/j.0001-7272.2005.00187.x.

Gavrilov IA, Kuznetsova VG. 2007. On some terms used in the cytogenetics and reproductive biology of scale insects (Homoptera: Coccinea). Comp Cytogenet. 1 , 169-174 DOI 10.1071/sp02002.

Gavrilov-Zimin IA. 2018. Ontogenesis, morphology and higher classification of archaeococcids (Homoptera: Coccinea: Orthezioidea). Zoosystematica Rossica, Supplementum. 2, 1-260.

Grabherr MG, Haas BJ, Yassour M, Levin JZ, Thompson DA, Amit I, Adiconis X, Fan L, Raychowdhury R, Zeng QD, Chen ZH, Mauceli E, Hacohen N, Gnirke A, Rhind N, Palma FD, Birren BW, Nusbaum C, Lindblad-Toh K, Friedman N, Regev A. 2011. Trinity: reconstructing a full-length transcriptome without a genome from RNA-Seq data. Nat biotechnol. 29, 644-652 DOI 10.1038/nbt.1883.

Gruwell ME, Hardy NB, Gullan PJ, Dittmar K. 2010. Evolutionary relationships among primary endosymbionts of the mealybug subfamily Phenacoccinae (Hemiptera:

Coccoidea: Pseudococcidae). Appl Environ Microb. 76, 7521-7525 DOI 10.1128/aem.01354-1.

Gullan PJ, Granston PS. 2014. The insects: an outline of Entomology (5th edition). Wiley Blackwell, Chichester.

Gullan PJ, Kosztarab M. 1997. Adaptations in scale insects. Annu Rev Entomol. 42, 23-50 DOI 10.1146/annurev.ento.42.1.23.

Hagan HR. 1951. Embryology of Viviparous Insects. Ronald Press, New York. 472. 
435 Hansen AK, Moran NA, 2014. The impact of microbial symbionts on host plant

436

437

438

439

440

441

442

443

444

445

446

447

448

449

450

451

452

453

454

455

456

457

458

459

460

461

462

463

464

465 utilization by herbivorous insects. Mol Ecol. 23, 1473-1496 DOI 10.1111/mec.12421.

Heinze J, Foitzik S, Oberstadt B, Rüppell O, Hölldobler B. 1999. A female caste specialized for the production of unfertilized eggs in the ant Crematogaster smithi. Sci Nat. 86, 93-95 DOI 10.1007/s001140050579.

Heinze J, Trunzer B, Oliveira PS. 1996. Regulation of reproduction in the neotropical ponerine ant, Pachycondyla villosa. J Insect Behav. 9, 441-450 DOI 10.1007/bf02214021.

Hombria JC, Brown S. 2002. The fertile field of Drosophila Jak/STAT signaling. Curr Biol. 12, 569-575 DOI: 10.1016/S0960-9822(02)01057-6.

Huang F, Zhao CL, Lu YB. 2015. Dynamics of Tremblaya princeps during individual development of its host Phenacoccus solenopsis (Hemiptera: Pseudococcidae). Acta Entomologica Sinica, 58, 1140-1145 DOI 10.16380/j.kcxb.2015.10.012.

Kosztarab M. 1996. Scale insects of northeastern North America: identification, biology, and distribution. Virginia Museum of Natural History Martinsburg, Virginia. 650.

Kranz B, Shibata T, Tsuchida K, Okajima S. 2002. Reproductive mode and split sex ratios in the facultatively ovoviviparous thrips, Bactrothrips brevitubus. Evol Ecol Res. 4, 1075-1092

Lagowska B, Golan K. 2009. Scale insects/Hemiptera, Coccoidea/as a source of natural dye and other useful substances. Aphids and other Hemipterous Insects. 15, 151-167.

Lee YH, Roger FH. 1987. Physiological roles of a yeast-like symbiote in reproduction and embryonic development of the brown planthopper, Nilaparvata lugens Stl. J Insect Physiol. 33, 851-860 DOI 10.1016/0022-1910(87)90033-3.

Lieber T, Jeedigunta SP, Palozzi JM, Lehmann R, Hurd TR. 2019. Mitochondrial fragmentation drives selective removal of deleterious mtDNA in the germline. Nature,570, 380-384 DOI 10.1038/s41586-019-1213-4.

Lloyd DC. 1952. Parthenogenesis in the mealybug Phenacoccus solani Ferris. Can Entomol. 84, 308-310 DOI 10.4039/Ent84308-10.

Lodé T. 2012. Oviparity or viviparity? That is the question. Reprod Biol. 12, 259-264 DOI 10.1016/j.repbio.2012.09.001. 
466 467 468

469

470

471

472

473

474

475

476

477

478

479

480

481

482

483

484

485

486

487

488

489

490

491

492

493

494

495

496

Ma H, Xu H, O'Farrell PH. 2014. Transmission of mitochondrial mutations and action of purifying selection in Drosophila melanogaster. Nat. Genet. 46, 393-397 DOI 10.1038/ng.2919.

McKenzie HL. 1967. Mealybugs of California with taxonomy, biology, and control of North American species (Homoptera: Coccoidea: Pseudococcidae). University of California Press, Berkeley, California. 526.

McGregor JR, Xi RW, Harrison DA. 2002. JAK signaling is somatically required for follicle cell differentiation in Drosophila. Development, 129, 705-717 DOI 10.1101/gad.213002.

Meier R, Kotrba M, Ferrar P. 1999. Ovoviviparity and viviparity in the Diptera. Biol Rev Camb Philos Soc. 74, 199-258 DOI 10.1017/S0006323199005320.

Nan GH Xu YP, Yu YW, Zhao CX, Zhang CX, Yu XP. 2016. Oocyte vitellogenesis triggers the entry of yeast-like symbionts into the oocyte of brown planthopper (Hemiptera: Delphacidae). Ann Entomol Soc AM. 753-758 DOI 10.1093/aesa/saw025.

Ngernsiri L, Piyajaraprasert W, Wisoram W, Merritt DJ. 2015. Structure of the female reproductive system of the lac insect, Kerria chinensis (Sternorrhyncha, Coccoidea: Kerridae). Acta Zool. 96, 312-318 DOI 10.1111/azo.12078.

Ortiz Rivas B, Moya A, Martinez Torres D. 2004. Molecular systematics of aphids (Homoptera: Aphididae): new insights from the long-wavelength opsin gene. Mol Phylogenet Evol. 30, 24-37 DOI 10.1016/s1055-7903(03)00155-6.

Ostrovsky, AN. 2013. From incipient to substantial: evolution of placentotrophy in aquatic colonial invertebrates. Evolution, 67, 1368-1382 DOI 10.1111/evo.12039.

Ostrovsky AN, Lidgard S, Gordon DP, Schwaha T, Genikhovich G, Ereskovsky AV. 2016. Matrotrophy and placentation in invertebrates: a new paradigm. Biol Rev. 91, 673-711 DOI 10.1111/brv.12189.

Tremblay E. 1997. Embryonic development, oviparity and viviparity. In: Ben-Dov, Y. and Hodgson, C.J., (Eds): Soft scale insects. Their biology, natural enemies and control. Elsevier Science, Amsterdam, The Netherlands. 257-260.

Tworzydlo W, Lechowska-Liszka A, Kocarek P, Bilinski SM. 2013. Morphology of the ovarioles and the mode of oogenesis of Arixenia esau support the inclusion of 
497 Arixeniina to the Eudermaptera. Zool Anz. 252, 410-416 DOI

$498 \quad 10.1016 / j . j c z .2012 .11 .002$.

499 Vennila S, Deshmukh AJ, Pinjarkar D, Agarwal M, Ramamurthy W, Joshi S,

500 Kranthi KR, Bambawale OM. 2010. Biology of the mealybug, Phenacoccus

501 solenopsis on cotton in the laboratory. J Insect Sci. 10, 1-9 DOI:

$502 \quad 10.1673 / 031.010 .11501$.

503 Warnecke U, Hintze-Podufal Ch. 1996. Sexual wing dimorphism in the oviviparous

504 cockroach Blaptica dubia (Blattoidea: Blaberoidea: Blaberidae). Entomol Gen. 20,

505 185-194.

506

Wheeler D. 2003. Reproduction. Female. In: Resh, W.H., Carde, R.T., editors.

507

508

Encyclopedia of Insects. Academic Press. 991-993.

Wilkinson TL, Ishikawa H. 2001. On the functional significance of symbiotic

509

microorganisms in the Homoptera: a comparative study of Acyrthosiphon pisum and

510

Nilaparvata lugens. Physiol Entomol. 26, 86-93 DOI 10.1111/j.1365-

511 3032.2001.00214.x.

512 Xie YP, Tian F, Liu WM, Zhang YF, Xue JL, Zhao YY, Wu J. 2014. The wax glands and wax secretion of Matsucoccus matsumurae at different development stages. Arthropod Struct Dev

.43, 193-204 DOI 10.1016/j.asd.2014.01.001.

Yin L, Wang K, Niu L, Zhang HX, Chen YY, Ji T, Chen GH. 2018. Uncovering the

516

517 changing gene expression profile of honeybee (Apis mellifera) worker larvae

518 transplanted to queen cells. Front Genet. 9, 416 DOI 10.3389/fgene.2018.00416.

519

Zhi FY, Huang F, Huang J, Li WD, LU YB. 2018. Biological characteristics of the

520 solanum mealybug, Phenacoccus solani (Hemiptera: Pseudococcidae). Acta Entomol Sinica. 61, 871-876 DOI 10.16380/j.kcxb.2018.07.014. 


\section{Figure 1}

The device used for the observation of egg-laying and egg hatching of adult females of Phenacoccus solani (Double-concavity slide method)

(A) Position and operation diagram. Two concave slides overlapped seamlessly, and adult female was placed into the middle of the hole of the concave slide; concave slides were fixed with rubber bands at both ends. (B) The final observation device contained two separate adult females. (C) Multiple devices together.

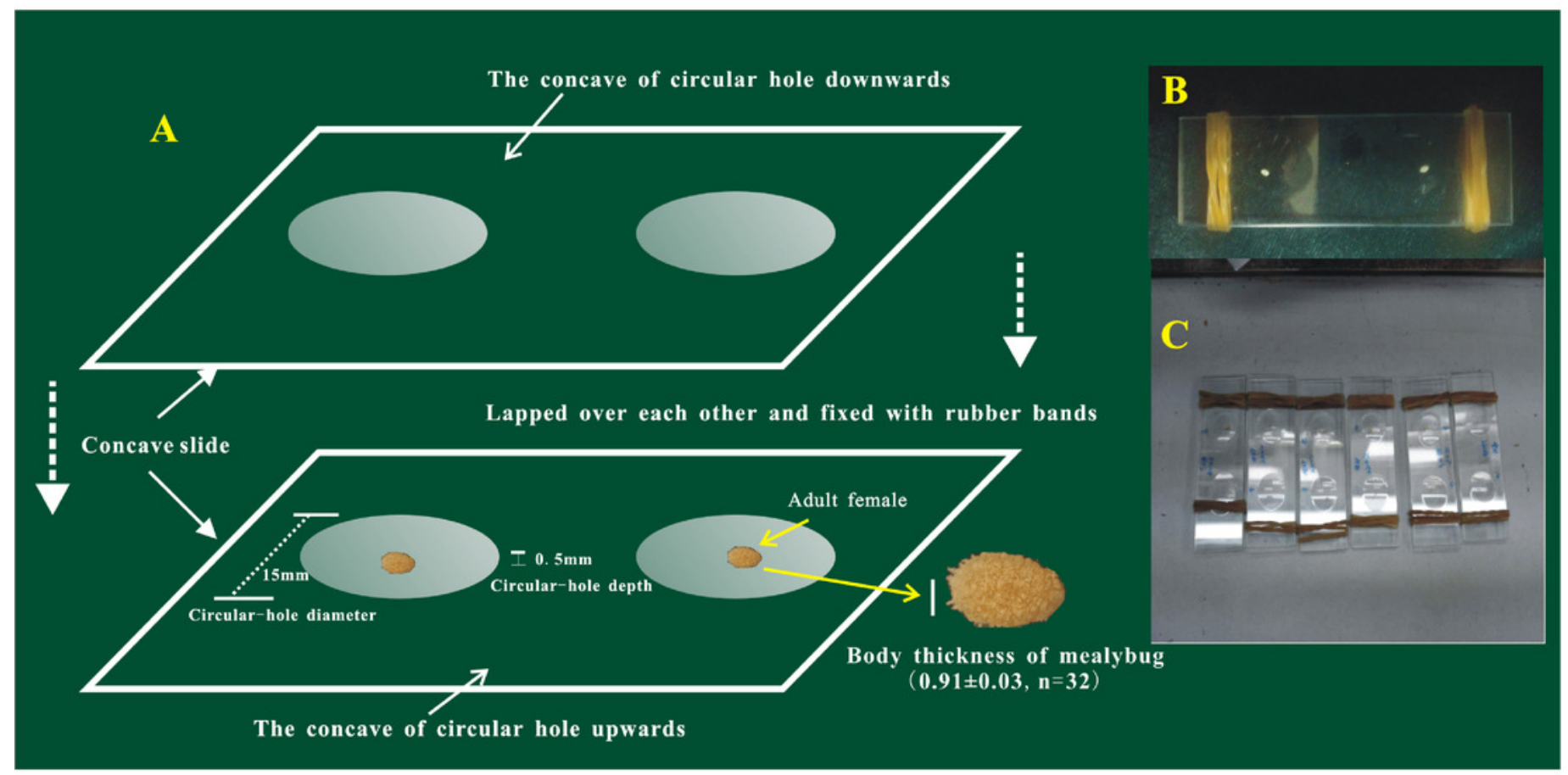




\section{Figure 2}

The hatching time of eggs beneath the abdomen of adult female or in isolation

(A) Egg hatching beneath the abdomen of adult female, at the point at which the eggshell detached; (B) Eggs with eyespots; (C) Egg hatching in isolation (without mother's body); (D) Hatching time of eggs beneath the abdomen of adult female; $(E)$ Hatching time of eggs without the mother's body or in isolation.
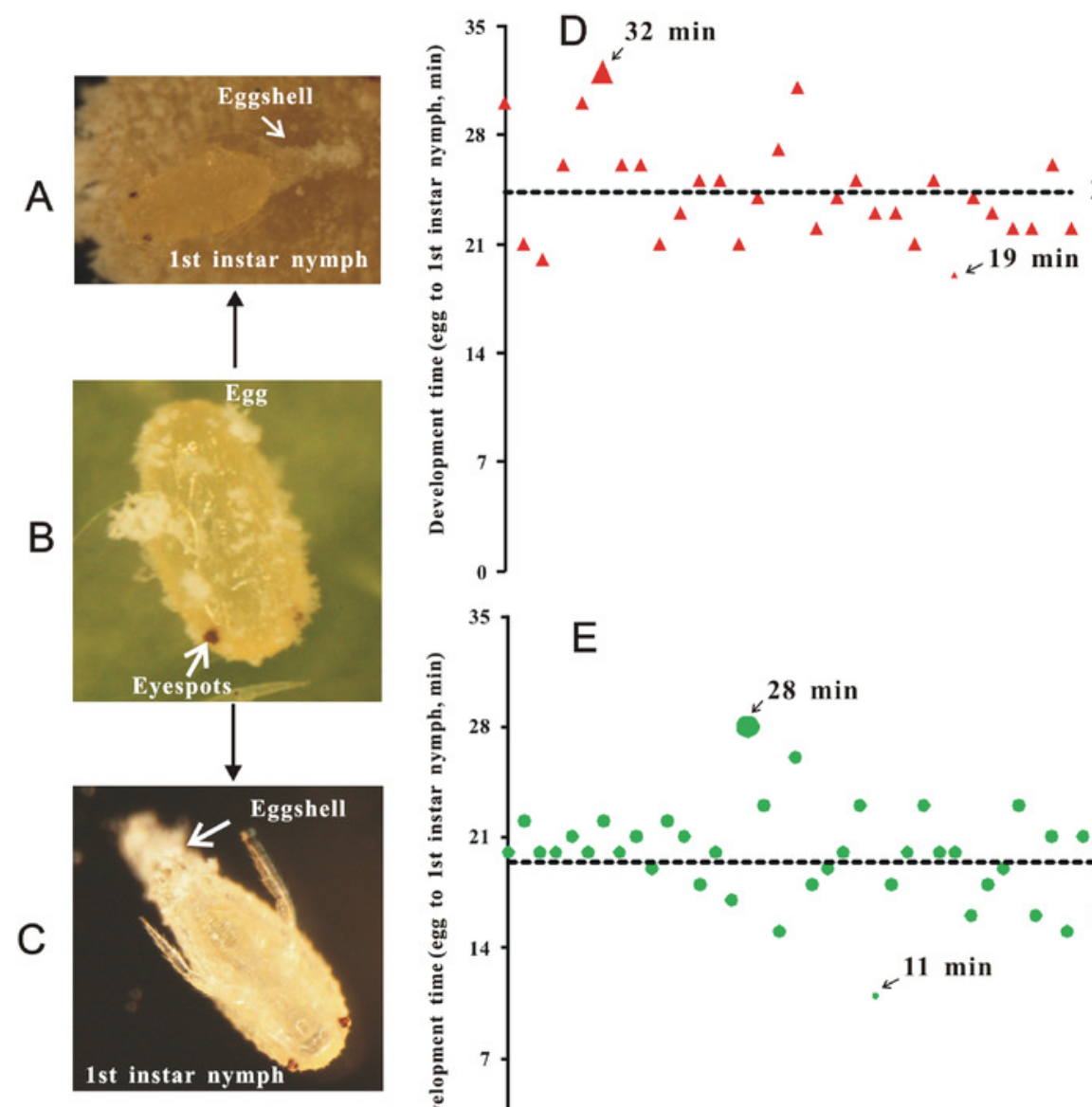

24. $30 \min ($ Average value, $n=30)$

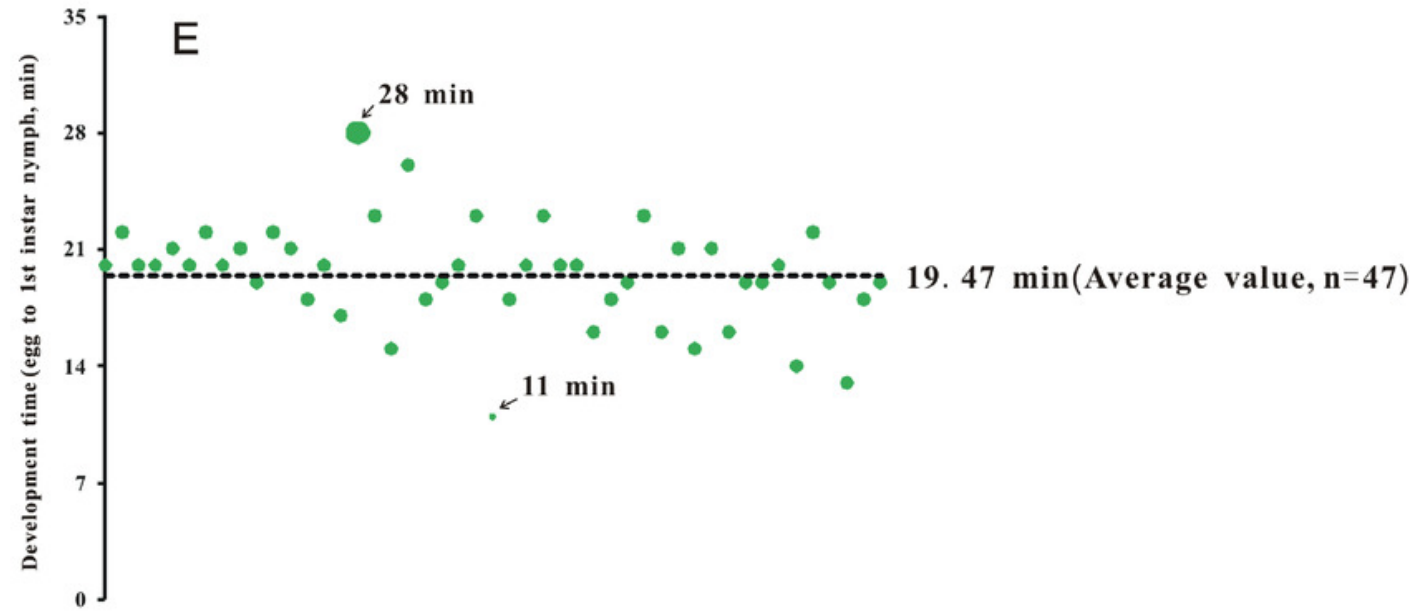




\section{Figure 3}

Adult female of Phenacoccus solani laid two types of eggs in one batch, one with eyespots (type 1 ), and another without eyespots (type 2 )

(A) Adult female laying eggs, and some eggs have rapidly hatched into 1st instar nymphs; (B) Newly laid two types of eggs below the abdomen of adult female; (C) The eyespots of eggs (type 2) were not visible; (D) Eggs (type 1) before hatching, the eyespots were clearly visible.

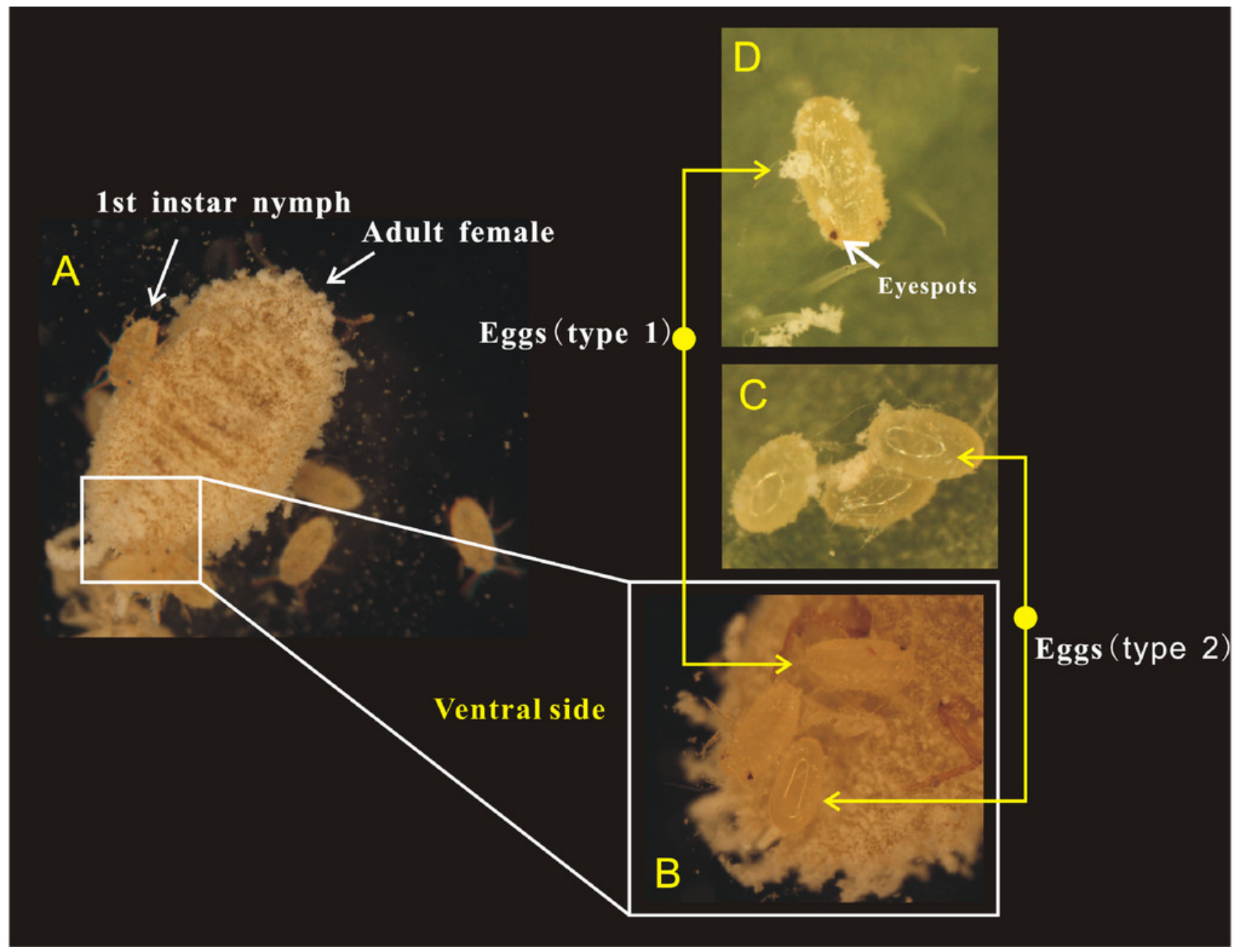




\section{Figure 4}

The hatching process of eggs

A) After the first 5 minutes, eggs began to show considerable peristalsis beneath the mother; B/C) At 10-15 minutes, the eggshell was gradually detached and wax powder appeared on the surface of body; D) At 20 minutes, antennae and feet were starting to become visible; E/F) The hatching was basically completed, while the detached eggshell could be seen at the abdominal end of the 1st instar nymph.
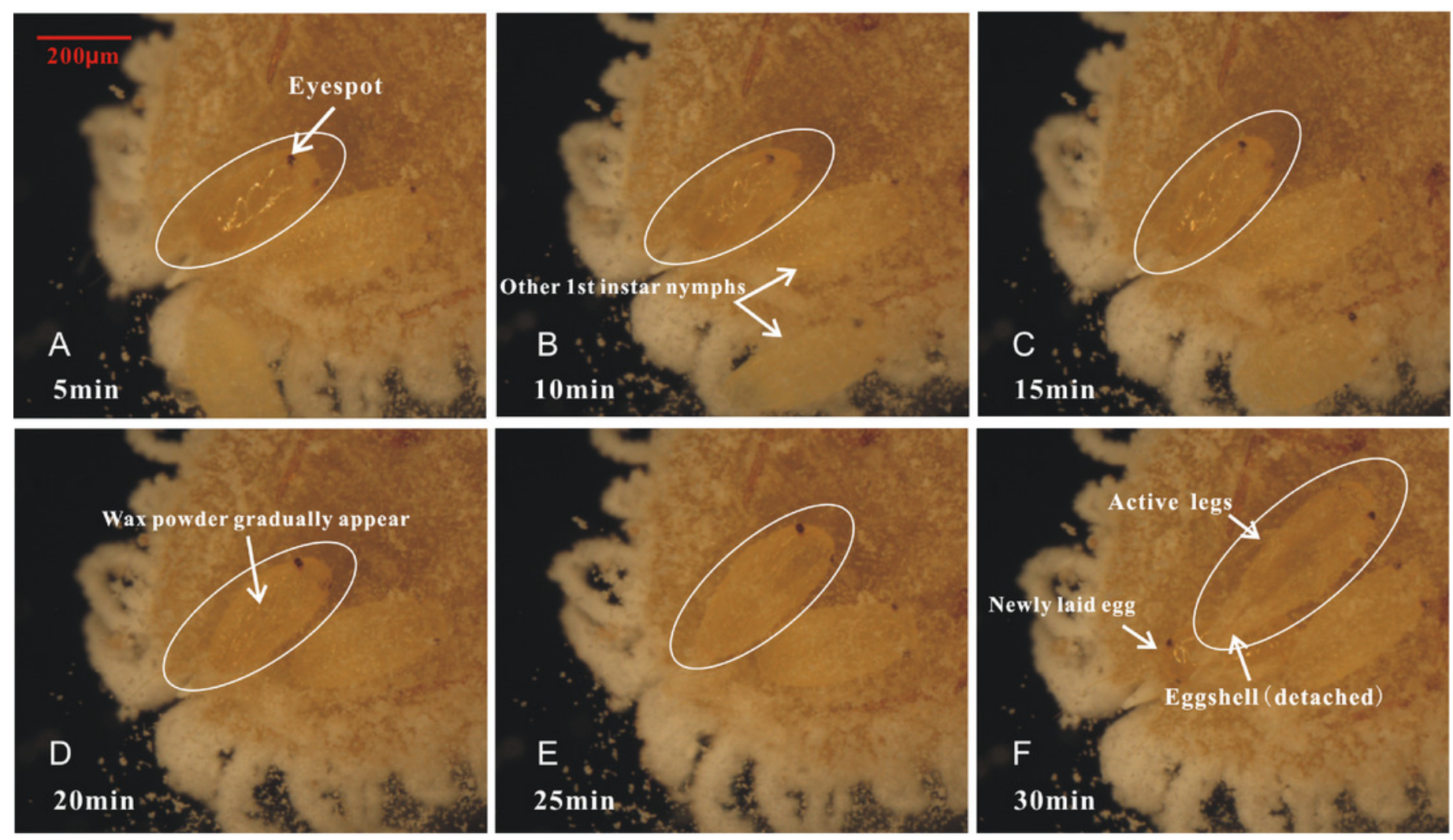


\section{Figure 5}

The percentage, morphology and hatching of the two types of eggs with different treatments

Percentage of the total for the two types of eggs with the treatment of placing female adults on leaves or concave slide (A), and hatching rate of eggs (B); the difference between the two types of eggs with same treatment was analyzed using Chi-SquareTest. The length (C) and width (D) of two types of eggs; the difference between the two types of eggs was analyzed using T-Test, and "NS" on the two bars indicate not significantly different from each other ( $p$ $>0.05$ ). 


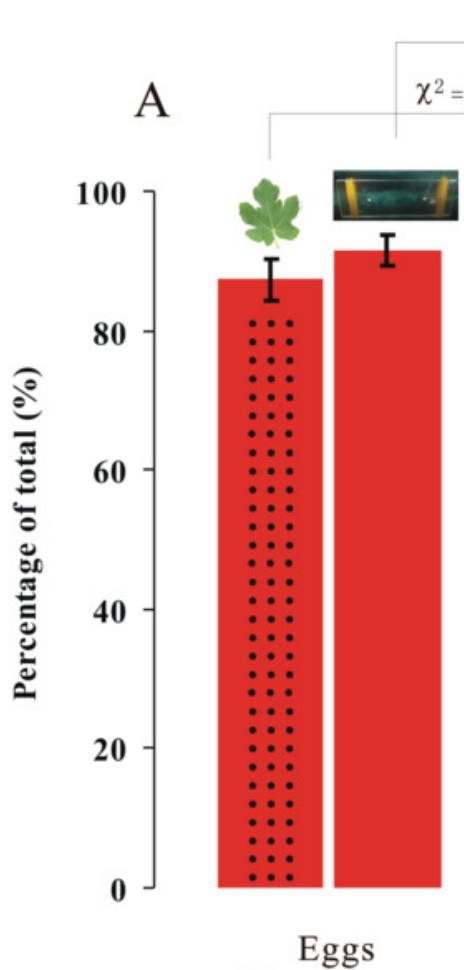

With eyespots

C $\quad(n=15)$

$\left.\begin{array}{cc} & 0.5 \\ & \\ & 0.4\end{array}\right]$ $\chi^{2}=86.01, P<0.001$

$=13.07, P<0.001$

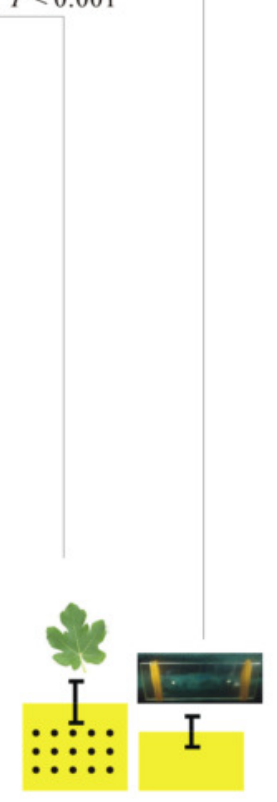

Eggs

Without eyespots

$(n=15)$
B

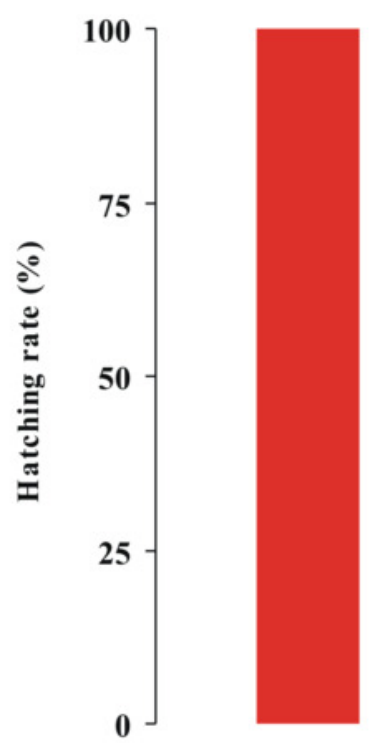

Eggs

With eyespots

D $\quad(n=114)$

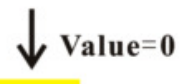

Eggs

Without eyespots

$(n=45)$

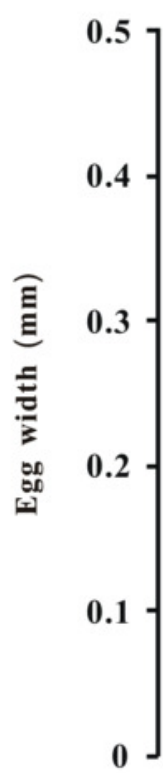

Eggs

Without eyespots

With eyespots $\quad$ Witho
\[ (n=25 \text { each }) \]

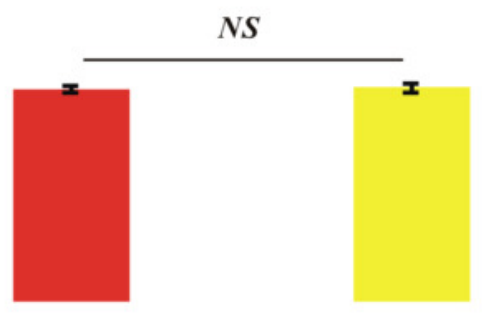

Eggs

With eyespots Without eyespots $(n=25$ each $)$ 


\section{Figure 6}

The surface and internal structure of eggs with eyespots and without eyespots

The surface of eggs with eyespots (A) and without eyespots (C) was observed by scanning electron microscope, and the internal structure of eggs with eyespots (B) and without eyespots (D) was observed by transmission electron microscope.

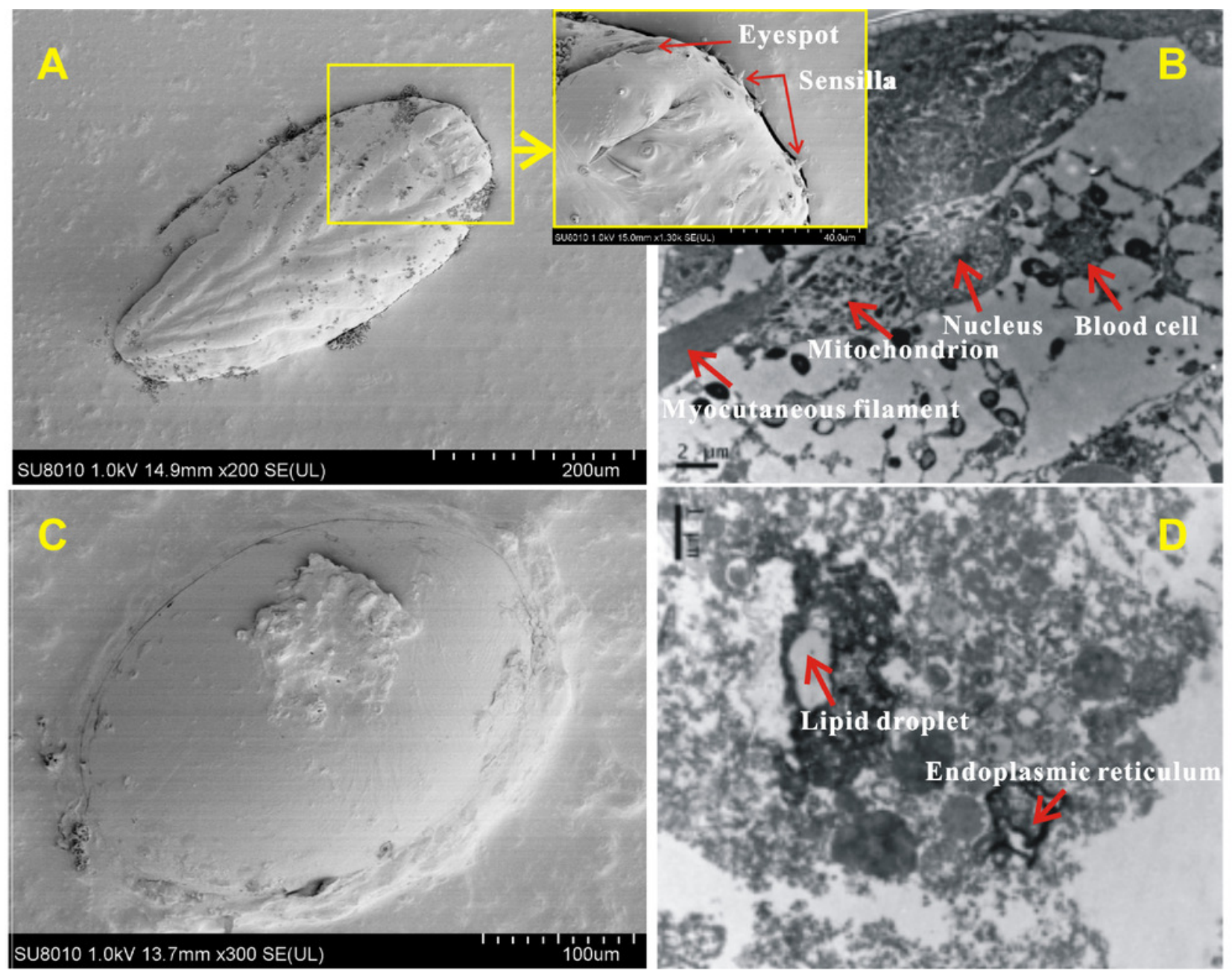




\section{Figure 7}

RNA-seq to distinguish the differences between two types of eggs.

Heat-map coefficient matrix (A); the abscissa indicates the sample name, the ordinate indicates the corresponding sample name, and the color indicates the correlation coefficient. The closer the correlation coefficient is to 1 , the higher the similarity in expression patterns between samples. Volcano plots of different differentially expressed unigenes (DEGs) between two groups. Green dots indicate down-regulated unigenes, red dots indicate upregulated unigenes, and grey dots indicate no differential unigenes (B). Cluster analysis of DEG levels. Expression differences are shown in different colors. Red and blue indicate upregulation and down-regulation, respectively (C). 

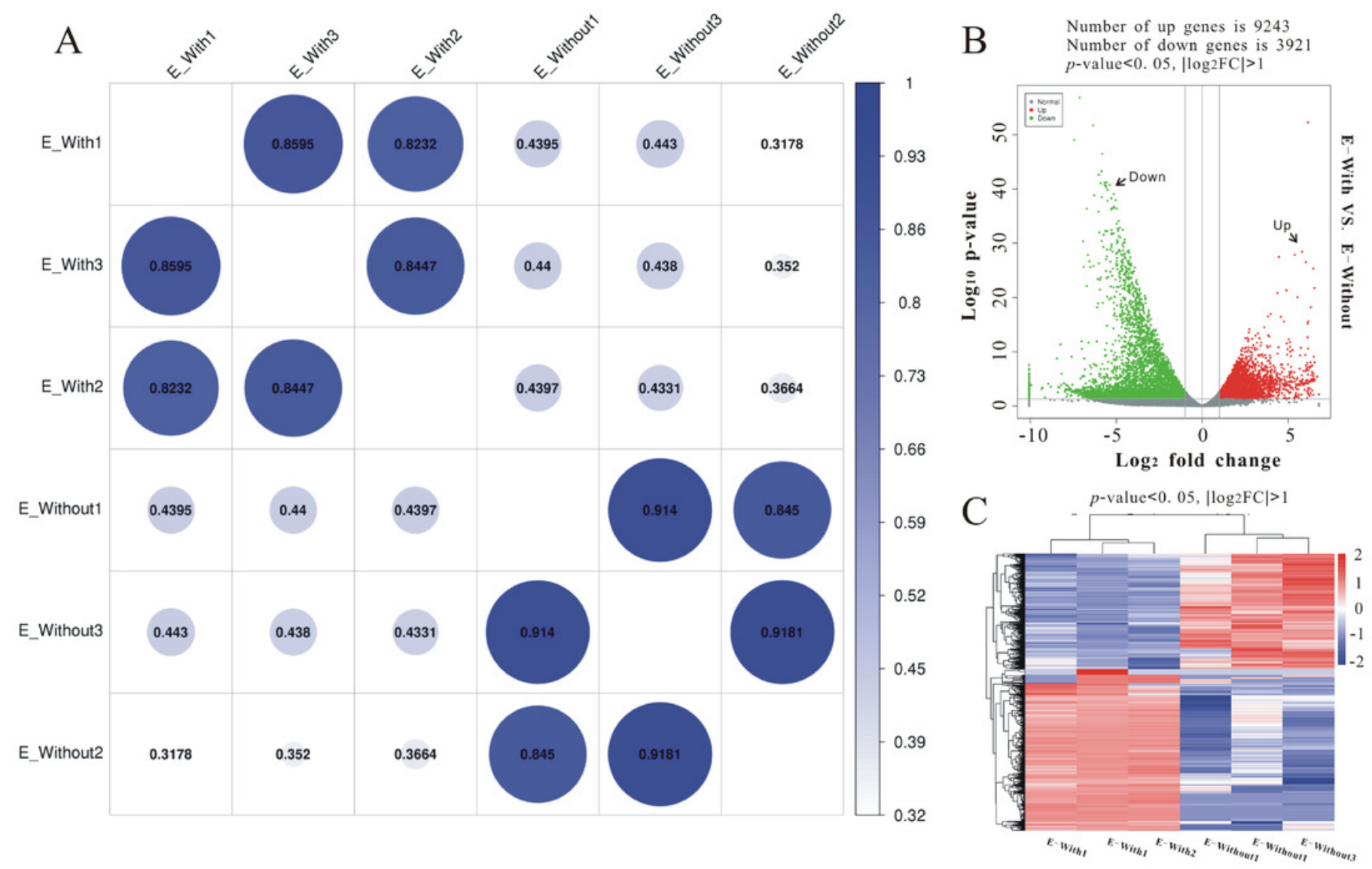


\section{Figure 8}

KEGG pathway enrichment analysis of differentially expressed genes (DEGs)

Only the top 20 pathways in KEGG enrichment function were listed in the figure. The ordinate is the name of the KEGG metabolic pathway, and the abscissa is the Enrichment Score to the pathway. The larger the bubble is, the more different the number of Unigene is contained, and the bubble color changes from purple-blue-green-red, with smaller Enrichment $p$ value and greater significance.

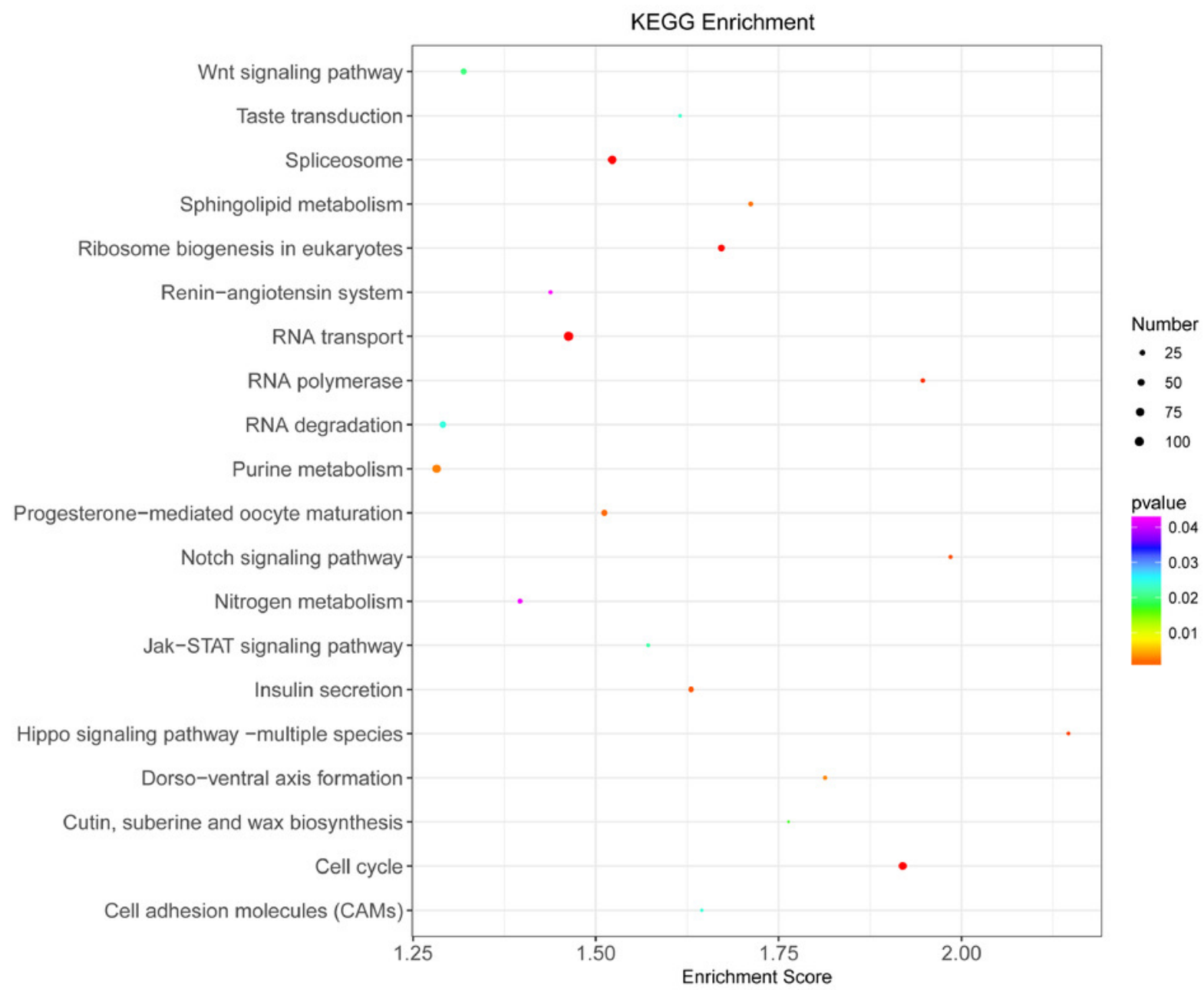

